\title{
Understanding intrinsic hematopoietic stem cell aging
}

\section{Eva Mejia-Ramirez ${ }^{1,2}$ and Maria Carolina Florian ${ }^{1,3}$}

${ }^{1}$ Center for Regenerative Medicine in Barcelona (CMRB), Bellvitge Institute for Biomedical Research (IDIBELL), Barcelona, Spain; ${ }^{2}$ Center for Networked Biomedical Research on Bioengineering, Biomaterials and Nanomedicine (CIBER-BBN), Madrid, 28029, Spain and ${ }^{3}$ Institute of Molecular Medicine and Stem Cell Aging, Ulm University, Ulm, Germany

$E M-R$ and MCF contributed equally to this work.

Volume 105(1):22-37

\section{Correspondence:}

MARIA CAROLINA FLORIAN

cflorian@cmrb.eu or

carolina.florian@uni-ulm.de

Received: July 14, 2019.

Accepted: November 14, 2019.

Pre-published: December 5, 2019.

doi:10.3324/haematol.2018.211342

Check the online version for the most updated information on this article, online supplements, and information on authorship \& disclosures: www.haematologica.org/content/105/1/22

(C)2020 Ferrata Storti Foundation

Material published in Haematologica is covered by copyright. All rights are reserved to the Ferrata Storti Foundation. Use of published material is allowed under the following terms and conditions:

https://creativecommons.org/licenses/by-nc/4.0/legalcode. Copies of published material are allowed for personal or internal use. Sharing published material for non-commercial purposes is subject to the following conditions:

https://creativecommons.org//icenses/by-nc/4.0/legalcode, sect. 3. Reproducing and sharing published material for commercial purposes is not allowed without permission in writing from the publisher.
ABSTRACT

$\mathrm{H}$ ematopoietic stem cells (HSC) sustain blood production over the entire life-span of an organism. It is of extreme importance that these cells maintain self-renewal and differentiation potential over time in order to preserve homeostasis of the hematopoietic system. Many of the intrinsic aspects of HSC are affected by the aging process resulting in a deterioration in their potential, independently of their microenvironment. Here we review recent findings characterizing most of the intrinsic aspects of aged HSC, ranging from phenotypic to molecular alterations. Historically, DNA damage was thought to be the main cause of HSC aging. However, over recent years, many new findings have defined an increasing number of biological processes that intrinsically change with age in HSC. Epigenetics and chromatin architecture, together with autophagy, proteostasis and metabolic changes, and how they are interconnected, are acquiring growing importance for understanding the intrinsic aging of stem cells. Given the increase in populations of older subjects worldwide, and considering that aging is the primary risk factor for most diseases, understanding HSC aging becomes particularly relevant also in the context of hematologic disorders, such as myelodysplastic syndromes and acute myeloid leukemia. Research on intrinsic mechanisms responsible for HSC aging is providing, and will continue to provide, new potential molecular targets to possibly ameliorate or delay aging of the hematopoietic system and consequently improve the outcome of hematologic disorders in the elderly. The nichedependent contributions to hematopoietic aging are discussed in another review in this same issue of the Journal.

\section{Introduction}

Aging is the largest risk factor for many chronic diseases and disabilities. Not surprisingly, aging is also the major risk factor for several hematologic syndromes and malignancies, such as myelodysplastic syndromes (MDS) and acute myeloid leukemia (AML). ${ }^{1}$ Moreover, aging has a negative impact on HSC regenerative capacity, and for this reason, cell-intrinsic mechanisms of aging are important putative targets for therapeutic interventions in order to ameliorate the consequences of aging on HSC and on the hematopoietic system. ${ }^{2}$ Understanding the mechanisms of HSC aging will provide the scientific community with new tools to improve the regenerative capacity of healthy HSC and thus the function of the hematopoietic system in the elderly.

The elderly population is growing rapidly worldwide. In addition, hematologic disorders and leukemia are exponentially growing with aging, without an equivalent acceptable growth in the therapeutic management of these diseases in the elderly; this is in sharp contrast to the increase in successful therapies for leukemia in younger patients. So far, with conventional induction therapy, many elderly patients experience a very poor overall survival rate, while requiring substantial social and medical assistance during their few remaining months of life, at a significant cost to the health service. ${ }^{3,4}$ 
A focussed understanding of the biology of aging in HSC and new therapeutic approaches is, therefore, mandatory.

\section{Intrinsic aging drivers}

Hematopoietic stem cells are the cornerstone of the hematopoietic system. Like other adult stem cells, they need to be localized in special niches that support and control the main stem cell functions: self-renewal and differentiation. Since HSC are so critical to the hematopoietic system and have to be functional during the entire life-span of the organism to maintain blood homeostasis, it is logical to think that somehow they require special protection from aging. Several studies have been trying to address how HSC can endure the effects of aging. However, investigating HSC function in living organisms is extremely challenging, since HSC constitute a rare cell population that, for most of the time, remain quiescent, undergoing very few divisions during the life-span of the organism (reviewed by Chandel et al..$^{5}$ and Singh et al. ${ }^{\circ}$. During aging, changes occur both in the niche, defined as extrinsic, and within the HSC, therefore dependent exclusively on the stem cell itself and, by definition, transplantable.

Working on human aging, especially in the hematopoietic system, has important experimental limitations and for this reason most of the research has been performed in model systems, particularly mouse models. Although some aging characteristics can be translated from mice into the human system, some phenotypes and their mechanistic investigation do not find a parallel in humans. Therefore, the development and establishment of possible new therapies must involve research on humans, or at least on models more similar to humans, such as nonhuman primates. Throughout this review, we discuss findings obtained in murine studies and we highlight some results obtained from studies carried out also in nonhuman primates and humans.

In general terms, a set of phenotypic and functional alterations have been consistently reported to characterize aged HSC (Figure 1).

\section{Increase in phenotypic HSC number and decrease regenerative capacity}

The number and frequency of HSC in the bone marrow of mice and humans increase with aging, while their regenerative capacity measured in transplantation assays is clearly reduced. It is accepted among researchers that this phenotype is caused primarily by cell-intrinsic mechanisms, ${ }^{7.9}$ even though an aged microenvironment can further aggravate it. . $^{1-12}$

\section{Myeloid skewing}

Aged HSC show an increased differentiation potential to the myeloid lineage and a decrease in the potential to differentiate into the lymphoid lineage. ${ }^{9,13,14}$ Moreover, even though the number of myeloid cells is higher, their quality is compromised. ${ }^{15,16}$ Interestingly, experiments dealing with single cell transplantations in mice $e^{17}$ and scRNA-sequencing (scRNA-seq) profiling ${ }^{18}$ have shown that HSC differentiation potential is not as homogenous as it was thought. In particular, "myeloid-restricted repopulating progenitors" (MyRP) have been characterized within the young HSC compartment. ${ }^{17}$ This MyRP subpopulation increases dramatically with age, in contrast with the moderate increase in the amount of multipotent HSC. ${ }^{17,18}$ Intriguingly, within this MyRP subpopulation, a subset of cells called "latent-HSC" can revert to the multipotent HSC state upon secondary transplants only in the aged samples. ${ }^{17}$ Consequently, it would be interesting to know if this subpopulation exists, and if it has the same behavior and features in the human bone marrow as in mice, in order to optimize transplantation therapies involving aged patients. These results highlight the potential importance of single cell profiling, at the molecular and at the cellular level, in order to better characterize also the human HSC compartment.

\section{DNA damage}

Accumulation of DNA damage is a common feature of aging in different tissues in many organisms, including humans. ${ }^{19}$ Although HSC have many mechanisms that enhance their capacity for protecting their genome from DNA alterations, ${ }^{20,21}$ some specific mutations have been shown to be highly recurrent in HSC..$^{10}$ In addition, upon aging, some of these somatic mutations are fixed and expand within the aged HSC compartment and are thought to be causally involved in the emergency of specific clones, which are the biggest contributors to replenishing the hematopoietic system. ${ }^{22}$ It is possible that some of these mutated clones could eventually progress to blood malignancies such as MDS or AML, although this is not necessarily the case. ${ }^{23}$

\section{Clonality}

In a young individual, hematopoiesis is normally supported by different HSC clones with similar potential. This has been demonstrated by limiting-dilution transplantation experiments. ${ }^{10}$ However, in the elderly, even though there is an increase in the number of HSC in the bone marrow niche, ${ }^{8}$ there is a smaller number of HSC clones that disproportionally contribute to the peripheral blood production (reviewed by Akunuru and Geiger²). This phenomenon is termed Clonal Hematopoiesis of Indeterminate Potential (CHIP) ${ }^{24}$ and is frequently detected in individuals over 55-60 years of age, being associated with an increased risk of hematopoietic malignancies and enhanced cardiovascular risk. ${ }^{25}$ In recent years, there has been increasing interest in studying how CHIP develops over time and eventually evolves into hematopoietic malignancies, given that this might prove useful in developing therapeutic approaches. ${ }^{23,26}$

\section{Epigenetic drift}

Specific changes occur in the epigenome and chromatin organization of aged HSC compared to young HSC. These changes include DNA methylation, ${ }^{27}$ specific histone posttranslational modifications, and chromatin reorganization. ${ }^{28}$

\section{Cell polarity}

Several molecules appear to be polar within the cell and the nucleus in young HSC when compared with aged HSC. For some of these molecules, both their polarity feature and the functional relevance are conserved from Drosophila to humans. In recent years, a few studies have demonstrated that the impairment in the function and stem potential of HSC upon aging are directly related to the loss of polarity of selected biomolecules within the cell. $12,15,29,30$ 


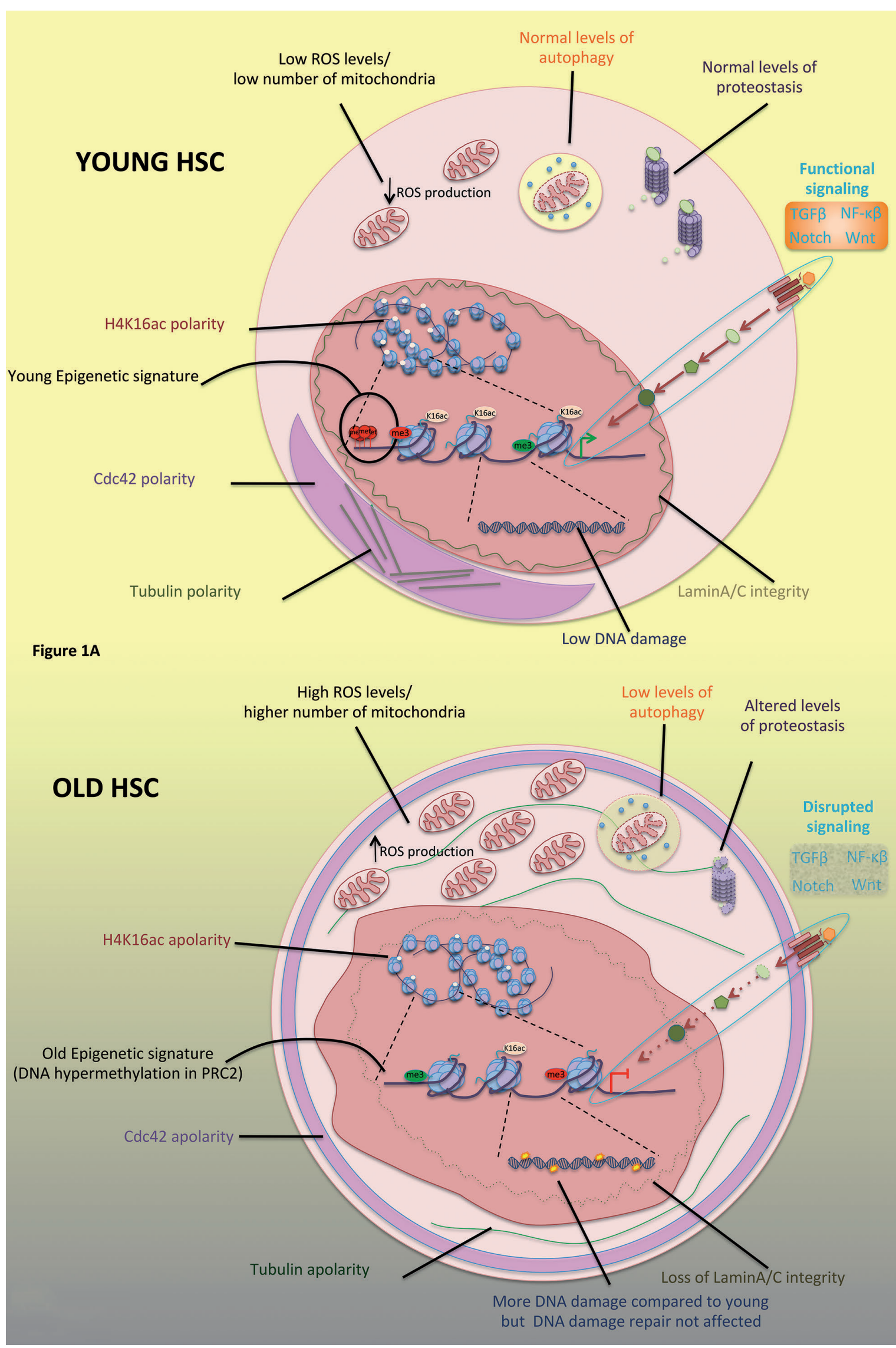

Figure 1. Intrinsic hallmarks of hematopoietic stem cells (HSC) aging. (A) Representative features of young HSC. (B) Representative features of aged HSC. K16ac: H4K16ac; met: DNA methylated; green me3: H3K4me3; red me3: H3K27me3. ROS: Reactive oxidative species; PRC2: Polycomb Repressive Complex 2. 


\section{Metabolic alterations and impaired autophagy}

Hematopoietic stem cells are characterized by having a low metabolic rate, being essentially glycolytic while quiescent. ${ }^{5,31}$ Upon activation, young HSC change towards a more oxidative metabolism that can be reverted when they return to quiescence (reviewed by Verovskaya et al..$^{32}$ ). However, in aged HSC, basal metabolism is shifted to a higher level of oxidative metabolism (reviewed by Verovskaya et al. ${ }^{32}$ ), which increases reactive oxidative species (ROS) leading to oxygen related-stress, accompanied by impaired regenerative potential (reviewed by Chandel et al..$^{5}$. Metabolic stress can fire autophagy in the cell, which is a "housekeeping" mechanism involved in self-degrading components of the cell in response to a specific stress. ${ }^{33}$ The process consists of enclosing organelles or portions of the cytosol within double membrane vesicles, which later fuse with the lysosome, where the degradation takes place. ${ }^{33,34}$ Autophagy deregulation has been related with aging and with age-related diseases such as cancer. ${ }^{34,35}$

\section{Altered proteostasis}

Proteostasis or protein homeostasis is described as the situation of balanced levels of protein biogenesis and degradation. ${ }^{36}$ Proteostasis is regulated by several mechanisms such as autophagy, the ubiquitination proteasome system (UPS), the unfolded protein control system (UPR), and the proper levels of protein translation. ${ }^{37}$ As mentioned above, autophagy is impaired with age, but also UPS and UPR levels are reduced leading to a situation of protein stress that contributes to loss of regenerative potential of aged HSC. ${ }^{36,38,39}$

\section{Alterations in intrinsic signaling pathways}

Signaling pathways such as TGF1- $\beta$, Notch, NF-кB or Wnt have an essential role in modulating the response of the hematopoietic system within the hematopoietic niche, tightly regulating the balance between quiescence and differentiation. ${ }^{40-43}$ These pathways are intrinsically altered in aged HSC, with a consequent effect on their function. ${ }^{15,28,44,45}$

The hallmarks of intrinsic HSC aging cover a very diverse group of biological characteristics that might nevertheless be all interconnected. Here, we will focus on some of these intrinsic molecular aspects, that are implied in driving functional and phenotypical alterations of HSC upon aging.

\section{Is DNA damage a driver of HSC aging?}

DNA damage and genomic instability are thought to be primary causes of mutation accumulation upon aging, leading to cancer or premature aging of tissues. ${ }^{19}$

Here we summarize what is known about the effects of DNA damage on aging of HSC under a different perspective, with consideration of new data generated by stateof-the-art techniques.

\section{From DNA damage to hematopoietic clonality}

There are premature aging syndromes, such as Werner or Bloom syndrome, in which the cause of the disease is the accumulation of mutations due to an inefficient DNA damage repair. The accumulated mutations in these syndromes lead to different kinds of cancer to arise at early age together with some premature aging phenotypes such as immunosenescence, skin fragility and osteoporosis. However, these syndromes recapitulate only some physiological aging characteristics, but not all. ${ }^{46}$

In the case of HSC, work performed on mice deficient in DNA repair proteins shows an expected reduction in HSC repopulation potential together with a decrease in their self-renewal capacity, driving a reduction of the whole HSC pool. ${ }^{31,47,48}$ As one of the most evident phenotypes occurring upon chronological aging is the expansion of HSC, the phenotype observed in mice deficient in DNA repair proteins sharply contrast with physiological HSC aging. More recently, it was elegantly shown by genetic barcoding and clonal functional evaluation of young and aged HSC followed by induced pluripotent stem cells (iPSC) generations and re-differentiation that, despite the heterogeneity of the aged HSC pool, several functional aspects of HSC aging could be reversed to a young-like state. These data strongly support the view that accumulations of DNA mutations in genes critical for hematopoiesis would not be the principal mechanism for the aged-dependent functional decline of $\mathrm{HSC},{ }^{49}$ although they might contribute to clonality. ${ }^{22,23}$

Still, mutations occur in every tissue and they are shown to accumulate with age. It has been calculated that somatic mutations in human HPSC occur and accumulate in life in a linear rate of $14 \mathrm{bp}$ base substitutions per year, which is similar to or even lower than other tissues..$^{50}$

$\gamma \mathrm{H} 2 \mathrm{~A}$ has been historically interpreted as being a marker of DNA damage in general, and in HSC in particular, since it is one of the first signals occurring at the damaged site and it is needed for subsequent DNA repair. ${ }^{48,51}$ Aged HSC present with increased numbers of $\gamma \mathrm{H} 2 \mathrm{~A}$ foci compared to young HSC..$^{48}$ Therefore, it was logical to conclude that there is deficiency in DNA repair in old HSC because of the persistence of $\gamma \mathrm{H} 2 \mathrm{~A}$. Interestingly, $\gamma \mathrm{H} 2 \mathrm{~A}$ foci have been demonstrated to be not only markers of double strand breaks (DSB). $\gamma \mathrm{H} 2 \mathrm{~A}$ also signals DNA replication stress (i.e. replication fork collapse) and it accumulates at rDNA in old quiescent HSC. Furthermore, in aged murine HSC, $\gamma \mathrm{H} 2 \mathrm{~A}$ cannot be removed by the specific phosphatase (PP4c) as efficiently as it is removed in young HSC because PP4c is mis-localized. ${ }^{21}$

Moreover, murine young and old HSC have been demonstrated to be equally efficient in repairing DNA under induced DNA damage. ${ }^{20,21,52}$ HSC make this possible by having the main DNA repair pathways attenuated while quiescent and reactivating them once they re-enter the cell cycle.$^{20}$ In addition, when young and old HSC are subjected to DNA damage, they lack G1-S arrest and their apoptosis rate increases in order to minimize the acquisition of mutations. ${ }^{52}$

In spite of the ability of HSC to avoid mutation accumulation through age, a series of point mutations have been found in humans to be predominant with age, as is the case of mutations in the epigenetic regulators DNMT3, TET2 and ASXL1, among others.53.56 These mutations appear frequently in healthy older individuals and they are also found over-represented in MDS and AML patients. ${ }^{23}$ It can be hypothesized that these mutations confer a competitive advantage that leads to clonal hematopoiesis. This is supported by some studies in the murine hematopoietic system in which the loss of Dnmt3a or Tet2 enhances the self-renewal potential of HSC. ${ }^{57}$ DNMT3a and TET2 are epigenetic modifiers: DNMT3s catalyze DNA methylation $(\mathrm{mC})$ and TET2 oxidizes $\mathrm{mC}$ to hydroximethyl-C, 
which leads to de-methylation of DNA (reviewed by Zhang et al. ${ }^{58}$ ). As has been demonstrated in mouse models, TET2 seems to contribute to both differentiation and self-renewal through gene activation and repression, and DNMT3a might contribute to the same functions by repressing HSC genes and generating substrates for TET2 action. ${ }^{58}$ The overall result is that both DNMT3 and TET2 co-operate to prevent activation of lineage-specific transcription factors in HSC. ${ }^{58}$ As for ASXL1, it seems that by binding to chromatin when mutated it can alter the epigenome, increasing the tendency towards CHIP or even leukemic transformation in mice..$^{59}$ Mutations in the human homologs of these genes have also been found to be related to clonal hematopoiesis and MDS. .0,61 $^{60}$

However, these mutations in humans do not necessarily involve the development of malignancy (although this might occur), probably in combination with other unknown events. ${ }^{23,62}$ In addition, MDS does not necessarily always lead to the development of AML. The combination of different mutations might be critical to allow the development of sub-clones with higher competitive advantage implied in the final establishment of MDS or AML. ${ }^{23}$

Telomere shortening is a special kind of DNA damage in which the low activity of telomerase or its absence makes the length of telomeres shorter after every division cycle and leaves the DNA ends unprotected, activating the DNA damage response and DNA repair pathways (reviewed by Behrens et al. ${ }^{63}$ ). Since telomeres become shorter every cycle, their length is intimately related to the life-span of a cell. Based on this assumption, there have been many attempts to understand if and how telomere length is related to life-span of the entire organism. Experimental observations indicate that the lower the rate of telomere shortening, the higher the life expectancy. ${ }^{64,65}$ As for HSC, it has long been known that they can only be serially transplanted 4-6 times in mice before they start showing loss of multi-lineage reconstitution capacity. ${ }^{66}$ This outcome was linked to limited replicative potential due to telomere shortening. However, it has been reported that HSC telomere length does not decrease in serial transplantation assays, ${ }^{67,68}$ and the overexpression of telomerase in mice does not lead to prolonged HSC transplantation capacity. ${ }^{67}$ The difference between mice and humans is remarkable in how the hematopoiesis system is affected by telomere attrition. For example, only the fifth generation on a Tert" mouse background develops anemia. ${ }^{69}$ This might be due to the elevated tolerance of murine HSC to accumulate alterations in their genome upon telomere shortening, either by entering in a senescence state or by undergoing apoptosis. ${ }^{70}$ In contrast, human patients of telomeropathies like Dyskeratosis congenita or adults with telomere gene mutations display very early bone marrow failure and severe aplastic anemia, ${ }^{71}$ which make the patients dependent on transplantation therapy.

Interestingly, a recent study with mice has shown that the loss of expression of Pot1a, a ssDNA binding protein part of the shelterin complex that binds telomeres, diminishes the potential of LT-HSC in vitro and in vivo, and its overexpression increases the contribution of LT-HSC to peripheral blood and bone marrow after secondary transplant, even rescuing the myeloid skewing in aged mice. ${ }^{72}$ Importantly, Hosokawa et al. included data showing that also the human homolog, POT1, improved ex vivo culturing of human cord blood HSC. ${ }^{72}$
Clonal hematopoiesis seems to stem out as a consequence of HSC mutation accumulation during aging (Figure 2). As has been shown in mice, the HSC compartment has a clonal dynamic nature that changes over time, with individual clones that expand or shrink, disappear or appear. ${ }^{26}$ However, there are differences between the results obtained in mice and those obtained in other model organisms, such as non-human primates. An extensive study dealing with the impact of aging on non-human primate hematopoiesis was recently published. It was demonstrated by lentiviral barcoding and high-throughput sequencing that aged macaques show a delay in the emergence of hematopoietic contribution in transplantations of autologous HSPC compared to mice, together with a persistent output from both B-cell and myeloid-biased clones. ${ }^{73}$ These results on clonal behavior of aged nonhuman primate HSC is of special importance for diagnosis and therapy in humans, since it is unfeasible to clonaltrack HSC in healthy patients. Mainly, information on human clonality and its progression upon aging comes from studies of "end-point" clonal population in healthy aged patients ${ }^{62}$ or by tracking existing clones from MDS patients to monitor their progression to AML over time. ${ }^{23}$ Importantly, a very interesting simulation tool has been recently reported in order to understand clonal contribution to hematopoiesis that can be applied to different species, from mice to humans. ${ }^{74}$

Under the premise that clonality is the result of specific mutations that give rise to "fitter" hematopoietic clones which are more successful after the selective process, one last question continues to be asked: does clonality result from intrinsic changes in aged HSC, or is it rather that clones are selected by the microenvironment ${ }^{275}$ Still, it is possible that the impairment of hematopoiesis upon aging might be the result simply of fewer contributing fit HSC which did not undergo intrinsic changes and which somehow resist microenvironment pressure.

In order to address clonal hematopoiesis and the predisposition to develop blood malignancies with age, many laboratories have tried to engineer AML mouse models. It has been shown that, for example, the deletion of Tet2, in mice leads to deregulated hematopoiesis and subsequent development of blood malignancies: ${ }^{76}$ however, the phenotype that is observed in aged human patients is not recapitulated completely in the murine models, either because of the onset of the disease, its penetrance, or the malignancy of the developed disease. ${ }^{6,77}$ New molecular biology approaches and a deeper understanding of the HSC aging process might be critical to improve the tools that are currently available. ${ }^{77}$

\section{Epigenetic changes: DNA methylation, histone marks and chromatin architecture}

Genome-wide expression profiling of murine young and aged HSC has shown that there are transcriptional alterations associated with aging in HSC which affect myeloid and lymphoid differentiation. ${ }^{728,43,78}$ Single-cell RNA sequencing (scRNAseq) has become a powerful tool to identify variations in transcriptional profiling between different cells from the same compartment, as in the case of HSC in the murine bone marrow. The information retrieved from scRNAseq from young and aged murine HSC has revealed that there is a molecular bias priming 
platelet genes expression at the expense of lymphopoietic genes, confirming the well-known myeloid skewing feature of aged HSC. 18,79

The suggested mechanisms underlying such a genomewide transcriptional change are related to the epigenomic regulation. Indeed, there is much evidence to support this hypothesis. For example, by reprogramming young and aged murine hematopoietic progenitors, the repopulation potential is indistinguishable between young and aged derived iPS into all hematopoietic lineages in chimera individuals and in HSC transplantations. ${ }^{49,78}$ These data imply that reprogramming is resetting the epigenetic state of the HSC regardless of the age at which the cells are reprogrammed. Moreover, these data also suggest that epigenetic alterations that occur upon aging are reversible..$^{00,81}$

The epigenomic landscape of HSC has been shown to change with respect to DNA methylation, histone modifications and chromatin architecture (Figure 2).

\section{DNA methylation changes from young to aged hematopoietic stem cells}

Global hypomethylation occurs in most somatic cells and in primary cells during aging. ${ }^{19,81}$ In contrast, the major- ity of DNA methylation signatures during HSC aging in mice are highly conserved. . $7,28,82$ However, the overall methylation change of aged HSC compared with young HSC is different depending on the study. The studies performed using reduce-representation bisulfite sequencing $(\mathrm{RRBS})^{27,28}$ showed a slight tendency to hypermethylation with aging. In contrast Taiwo et al. reported a slight but significant $(5 \%)$ global loss of DNA methylation by MeDIP-seq. Taiwo et al. argue that the difference could be explained by the difference in coverage of each technique. Bisulfite-sequencing provides generally $5-10 \%$ genome coverage, in contrast with $60 \%$ provided by MeDIP-seq. ${ }^{82}$

Interestingly, independently from the overall hypo- or hypermethylation detected, all studies so far report high similarities in the alterations at specific loci that gained or lost methylation. One of the clearest examples involves polycomb repressive complex (PRC2) target genes, which are hypermethylated in aged HSC. ${ }^{27,82}$ It has been demonstrated in a human cell line that PRC2 cannot bind to chromatin if the DNA is hypermethylated..$^{33}$ Moreover, some PRC2 complex subunits in aged and proliferative stressed HSC resulted in being down-regulated as measured by RT-qPCR ${ }^{27}$ and RNA sequencing. ${ }^{28}$ These observations

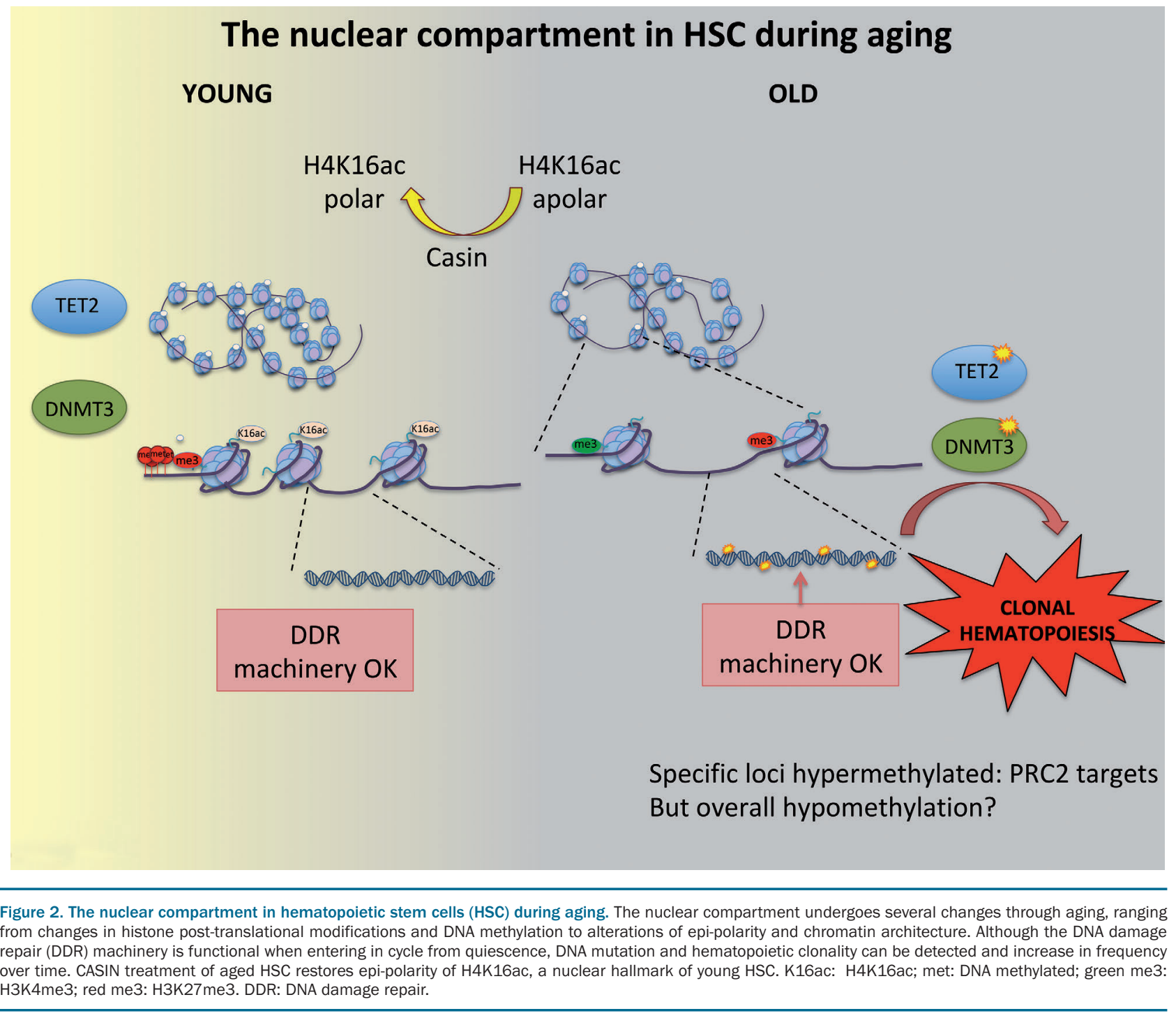


might suggest that the methylation machinery in old HSC might access PRC2 target sites merely as a consequence of the reduced levels of PCR2 itself, which normally occupies these specific targets inducing their transcriptional downregulation. However, this hypothesis has yet to be demonstrated.

A possible explanation for the limitation to a more global and prominent hypomethylation on HSC during aging is linked to their quiescence during adult life. When murine young or old HSC are transplanted, and are therefore subjected to an induced proliferative stress, global hypomethylation occurs. ${ }^{27}$ This observation agrees with reports for aging in human somatic cells and tissues (reviewed by Gonzalo ${ }^{81}$ and Jones et al. ${ }^{84}$ ). However, even in this case, the hypermethylation of PRC2 targets is still detectable in mice, suggesting that aging and exhaustion promote distinct alterations on the DNA methylation landscape..$^{27}$

It is worth noting that differentially methylated genes in aged HSC compared to young HSC are expressed or highly expressed in progenitor cells downstream in murine hematopoietic differentiation. ${ }^{27}$ This observation suggests that the impact of alterations in DNA methylation during aging is not probably manifested until HSC differentiate, and never in HSC themselves. This aspect is also demonstrated by the work of Scadden's team, who elegantly showed in mice that the differences in enhancer methylation between distinct HSC clones in the same individual has no effect on gene expression until stem cells differentiate into subsequent hematopoietic sub-compartments. ${ }^{26}$

\section{Histone post-translational modifications upon hematopoietic stem cell aging}

A number of histone tail modifiers have been involved in HSC function and also have a role in chromatin remodeling, which supports the concept that changes in the chromatin state within the HSC compartment are important for blood homeostasis in both mice and human systems. Among them, we can find PRC2 subunits such as Ezh2, Suz12 and Eed, ${ }^{85-87}$ histone specific lysine-demethylases such as Jarid1b/KDM5b ${ }^{88}$ UTX/KDM6a ${ }^{89}$ or Lsd1/KDM1a, ${ }^{90}$ or histone methyltransferases such as SUV39H1. ${ }^{91}$

Moreover, it is known that the expression levels of some of these proteins change upon aging, ${ }^{27,28,43,91}$ suggesting that histone modifications are different in old HSC when compared to young HSC.

Goodell's group performed one of the most accurate studies of epigenomic modifications in murine HSC upon aging. Some of the principal regulatory histone marks were profiled by ChIP-seq in HSCs from 4-month old and 24month old mice. Although the dataset revealed only moderate changes, some interesting unique features were reported. For example, regarding $\mathrm{H} 3 \mathrm{~K} 4 \mathrm{me} 3$, there was only an increment of $6.3 \%$ in peak deposition in old versus young HSC, but most of the peaks were generally broader.

As for H3K27me3, peak counts remained similar but an increase in the length of coverage by $29 \%$ was detected upon HSC aging. Moreover, the intensity of the signal increased by $50 \%$. In relation to $\mathrm{H} 3 \mathrm{~K} 36 \mathrm{me} 3$, the peaks moved from the TSS to the transcription termination site, and H3K36me3 and H3K27me3 behaved in a mutually exclusive manner. A strong positive correlation between transcriptome changes and the three histone marks analyzed in the study was also reported. ${ }^{28}$
Regarding other histone marks, H4K16ac levels and localization have been demonstrated to change with age in murine HSC. H4K16ac shows a prominent distribution in one pole of the nucleus in young HSC by single cell 3dimensional (3D) immunofluorescence analysis. This characteristic is defined as "epigenetic polarity" or "epipolarity". Old HSC show reduced levels of H4K16ac, which also displays apolarity and localizes evenly all over the nucleus. ${ }^{14,15,29}$ The polarity and levels of several other histone marks such as H3K4me1, H3K4me3, H4K8ac, H3K27ac and H4K5ac showed no aged-dependent changes in murine HSC and ST-HSC. ${ }^{29}$ Interestingly, the loss of epipolarity and the reduction of global H4K16ac deposition upon aging in HSC did not primarily correlate with global changes in gene expression. ${ }^{15} \mathrm{H} 4 \mathrm{~K} 16 \mathrm{ac}$ was associated to the modulation of higher-order chromatin and protein interactions between chromatin and non-histone proteins, ${ }^{92}$ and was shown to be targeted by inhibiting Cdc42 activity and LaminA/C expression. H4K16ac role in chromatin architecture in HSC will be further discussed below.

In the context of human AML, H4K16ac levels seem to be linked to the nuclear localization of PAK4, a target of the orphan gene C3orf54/INKA1, which promotes selfrenewal by inhibiting differentiation of cord blood HSC. ${ }^{93}$ C3orf54/INKA1 seems to be up-regulated in leukemic stem cells in AML patients, while PAK4 is an effector of Cdc 42 in humans. ${ }^{94}$

Altogether, the results obtained over the last decade suggest that aging has an effect on histone mark landscape in HSC. However, the specific alteration of histone marks upon aging not always correlates with global change on gene expression in HSC themselves, but it rather affects the expression of genes in downstream progeny during the differentiation program, as we stated above also for the methylation profile. ${ }^{15,26,27}$ Altogether, this makes epigenetic studies extremely cumbersome in HSC and subtle differences within this cell population might indeed make a functional impact visible only under specific challenges.

\section{Chromatin architecture changes}

Over recent years, chromatin architecture has become more important for gene expression, cell division, fate determination, growth and development, disease and even genome evolution (reviewed by Kong and Zhang ${ }^{95}$ ). Recent studies have proposed the existence of two different but related ways of $3 \mathrm{D}$ chromatin organization: the cohesin-dependent CTCF loops and the small compartmental domains that are formed as a consequence of transcription and chromatin state (reviewed by Rowley and Corces $\left.^{9}\right)$. In this regard, and in relation to HSC biology, some disease-associated mutations in humans that affect proteins involved in establishing or preserving chromatin architecture have been described, for example, proteins belonging to the cohesin complex, such as Stag2, Rad21 and Smc3, which have been associated with AML and MDS particularly in the elderly. ${ }^{97,98}$

Chromatin accessibility changes upon HSC aging have just started to be investigated. Recently, we performed single-cell ATAC-seq on HSC daughter pairs from young and old mice. The results showed that there was a greater difference between young pairs, more "asymmetrical", as based on quantity of ATAC-seq peaks, than those coming from aged pairs, which were, therefore, more "symmetrical". This observation was correlated to H4K16ac distribu- 
tion in daughter cells that was more asymmetric in young compared to old pairs. Signature-based, daughter stem cells were in general associated to an overall lower amount of ATAC-seq peaks, suggesting a progressive increase in chromatin accessibility upon differentiation. ${ }^{15}$ Interestingly, both young and aged daughter stem cells preserved open chromatin regions linked to glycolysis and small RhoGTPases signatures. Aging was found to impact prominently on the specific signature of open regions associated to critical signaling pathways in HSC (Wnt signaling was associated with aged HSC, while young cells were enriched for VEGF, TGF $\beta$ and EGF signaling). In addition, daughter progenitors maintained signatures linked to lipid metabolism and platelet homeostasis in both young and aged cells, while mostly they differed again for open regions linked to specific signaling pathways (interferon, IFG1 and IL2 were characterizing young cells and TNF was enriched in aged cells). ${ }^{15}$

Specific chromatin architectural alterations affecting broader alterations have also been detected in aged murine HSC, such as the relative position of chromosomes. We have observed that chromosome 11 homolog proximity changes upon aging specifically in HSC. This chromosomal rearrangement is dependent on LaminA/C expression and Cdc42 activity. ${ }^{29}$ Indeed, we demonstrated that upon aging and in chronologically young LaminA/C knock-out HSC, chromosome 11 homolog proximity was altered and this correlated with the functional impairment of stem cells. Inhibiting Cdc42 activity could restore LaminA/C expression and localization, and also chromosome 11 homolog proximity. Overall, these findings support the functional relevance of chromatin architecture also for HSC aging and, most importantly, show for the first time that is possible to pharmacologically target the chromatin architecture to rejuvenate aged HSC function. ${ }^{29}$

\section{Aging and the epigenetic drift}

Epigenetic drift is the term that covers all changes that have a general effect on the epigenome and the chromatin architecture..$^{99,100}$ Recently, an exciting report of the epigenetic drift in murine aging across tissues has been published. Benayoun et al. showed that the aged-related alterations observed in the epigenome and transcriptome landscape lead to induction of inflammatory responses, mainly interferon related, across different tissues upon aging. ${ }^{100}$ In humans, the epigenetic drift has been studied at the single-cell level on peripheral blood mononuclear cells from subjects of different ages. ${ }^{101}$ In agreement with previous studies performed on bulked murine HSC, ${ }^{27,28}$ they observed an increment of H3K4me3 and H3K27me3 with age. These variations were reflected in transcriptional alterations and an association of $\mathrm{H} 3 \mathrm{~K} 4 \mathrm{me} 3$ with DNA breakage and translocation was also observed. ${ }^{102}$

The fact that the observed epigenetic drift is consistent between mice and humans suggests that it is a conserved phenomenon in mammals. However, its ultimate manifestation seems very heterogeneous, even within cells and tissues of the same individual. This has been demonstrated using different approaches such as the generation of a multi-color Hue mouse model as a clonal tracking too ${ }^{26}$ and single-cell transcriptomics to study the transcriptional behavior of each clone. ${ }^{26,103}$ These two studies agreed in identifying (based on transcriptome analysis) distinct clones in aged individuals that have different regulatory states as a consequence of the epigenetic drift occurred upon aging. ${ }^{26,103}$

\section{Metabolism and hematopoietic stem cell aging}

Hematopoietic stem cells are quiescent during most of their lifespan and their metabolic needs are relatively low. However, one of the aging hallmarks is the dysregulation of metabolic pathways, and this aspect might indeed be critical for HSC function, too. Here we detail some of the key metabolic aspects related to HSC aging (Figure 3).

Effects of oxidative stress and mitochondria homeostasis in hematopoietic stem cell aging

It has been shown that artificially altering mitochondrial function can lead to acquiring aging phenotypes in HSC, such as loss of self-renewal and lymphoid/myeloid skewing. Sometimes these mitochondrial-related aging phenotypes can be reverted by the addition of redox scavenger compounds [N-acetylcysteine (NAC), rapamycin or MAPK inhibitors]. Normally, rapamycin and calorie restriction can only partially rescue the effects of aging due to intrinsic deteriorated mitochondrial activity (reviewed by Chandel et al. ${ }^{5}$ ).

Reactive oxygen species (ROS) are the natural sideproduct of oxygen-based metabolism and are produced mainly by mitochondria. The effect of ROS in aging has been historically attributed only to the oxidation of DNA, RNA and proteins, but it has an important role in cell signaling, too. ROS can be detected by redox sensors that ultimately fire oxidative stress response such as enzymes and transcription factors (reviewed by Bigarella et al. ${ }^{104}$ ).

In HSC, high levels of ROS can generate oxidative stress and lead to loss of quiescence in the cells; therefore, ROS levels have to be tightly controlled (reviewed by Bigarella et al. ${ }^{104}$ ). Quiescent HSC have a low metabolic rate, which involves low levels of reactive oxygen species (ROS) ${ }^{105}$ It has been shown in mice that the frequency of HSC with low levels of ROS decreases with age, suggesting that ROS generation/accumulation is a distinctive characteristic of aging. ${ }^{106}$ Increments of ROS levels in adult HSC have consequences that are somewhat similar to the aging phenotype such as myeloid lineage skewing and defective long-term repopulation activity, as has been demonstrated in the case of FoxO transcription factors depletion in mice. ${ }^{107,108}$ It is very interesting that high levels of ROS in the FoxO depleted HSC can be rescued by the addition of the antioxidant NAC, ${ }^{108}$ but only for some weeks. ${ }^{107}$ The importance of FoxO3 in LT-HSC function goes beyond regulation of ROS levels since it is essential for activation of the autophagy gene program. ${ }^{109}$

Reactive oxygen species are also augmented in HSC of aged SIRT3 knockout (KO) mice, negatively affecting their function, but not in young SIRT3 $\mathrm{KO}$ mice. Intriguingly, overexpression of SIRT3 rescues functional aged-related defects in HSC. SIRT3 is a deacetylase that regulates mitochondrial protein acetylation in mammalian cells and it is enriched in young compared to aged HSC. Therefore, the role of SIRT3 in the maintenance of proper ROS levels in HSC is essential during aging. ${ }^{110}$

Mitochondrial homeostasis refers to the correct regulation of the mitochondria function within the cell. Studies using "mutator mice" (proof reading mutant for Pol $\gamma \mathrm{A}$, the catalytic subunit of mtDNA polymerase that is encoded in the nuclear genome) showed phenotypes indicative of 
premature aging, ${ }^{111}$ including features of MDS. ${ }^{112}$ Trifunovic et al. concluded that there might be a causative link between mtDNA mutations in these mice and the aging phenotype. ${ }^{111}$ However, deep insights in the phenotype and molecular signatures of aged HSC demonstrated that they are different from this 'mutator' strain, concluding that aging is primarily independent of the accumulation of mitochondrial mutations. ${ }^{105}$ What is, indeed, observed is that aged HSC show deregulation that deteriorates mitochondrial activity (reviewed by Oh et al. ${ }^{113}$ ).

Maintenance of mitochondrial homeostasis within the cell is also dependent on the regulation of nuclear mitochondrial gene expression. For example, Mohrin et al. showed that SIRT7, a histone deacetylase, is normally located at the proximal promoters of ribosomal proteins and mitochondrial transcription factors within the nuclear genome. It also binds directly to NRF1, the master transcription factor of mitochondria. Eventually, SIRT7 leads to the inhibition of the expression of mitochondrial proteins in young HSC. ${ }^{114}$ Aged HSC have lower levels of SIRT7, thus allowing an increment in the expression of mitochondrial proteins. This, in turn, activates the PFSmt (mitochondrial protein folding stress), a metabolic checkpoint that reduces HSC quiescence. Therefore, overexpression of SIRT7 in aged murine HSC was shown to decrease the levels of mitochondrial protein expression associated with mitochondrial stress and to improve aged HSC function. ${ }^{114}$

\section{Autophagy}

Autophagy is a major regulator of mitochondria homeostasis and a suppressor of the metabolism. It is regulated by nutrient-sensing pathways like mTOR or AMPK, which inhibit and activate autophagy, respectively. In general, autophagy decreases with aging, due to either the downregulation or the upregulation of critical autophagy proteins. In HSC, the age-related decrease in autophagy has been associated with lower capacity of glucose intake; mitochondrial autophagy would be attenuated in order to maintain necessary energy levels in old HSC and is rapidly induced by FoxO3 transcription factor when HSC are under metabolic stress. ${ }^{109}$

Genetic tools were instrumental in revealing the critical role of autophagy upon HSC aging. ${ }^{115}$ For example, in

\section{Metabolic homeostasis and proteostasis during aging in HSC YOUNG OLD}

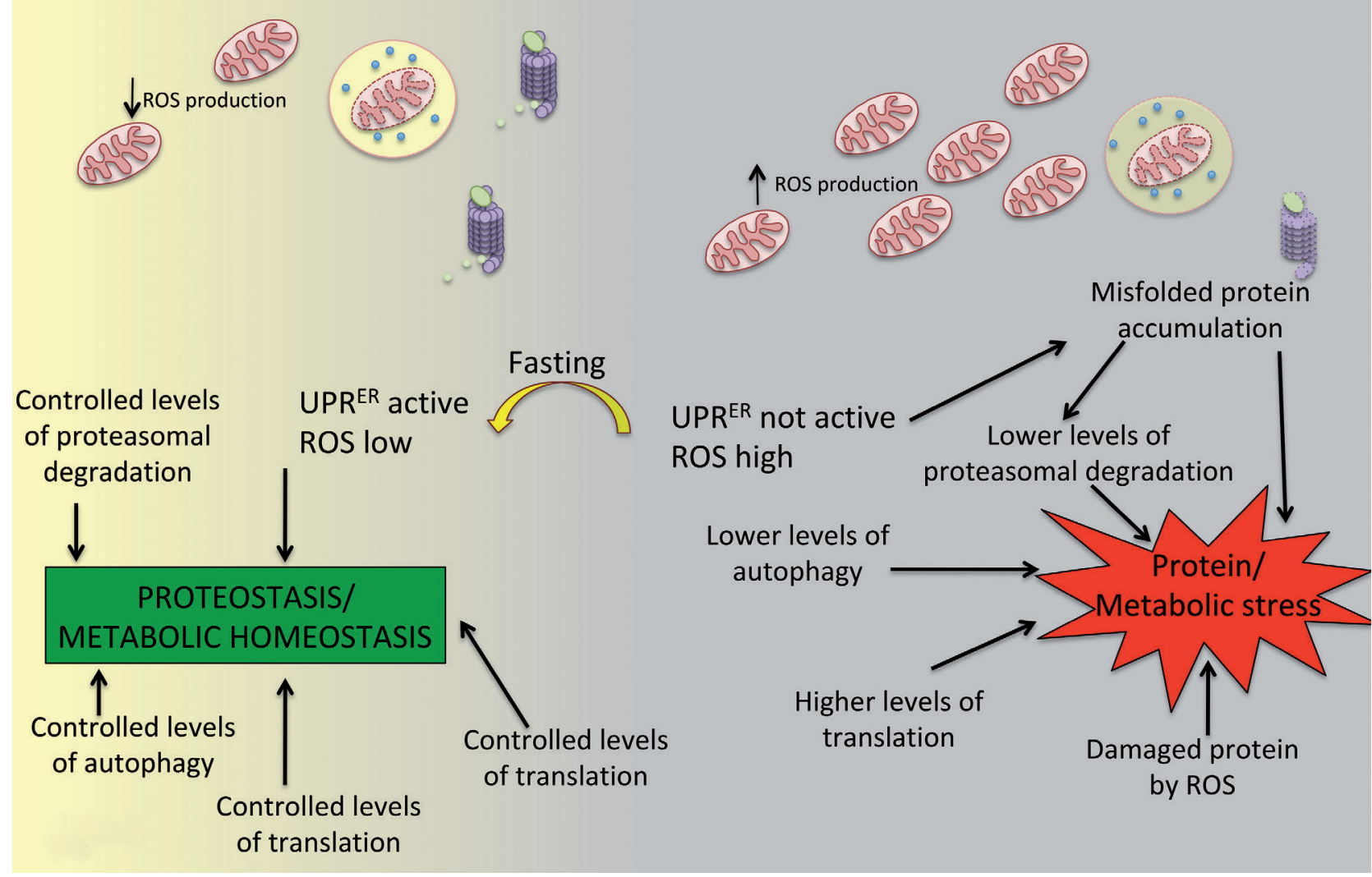

Figure 3. Metabolic homeostasis and proteostasis during aging in hematopoietic stem cells (HSC). Young HSC fine-tune several biological processes: they maintain a low metabolic rate, control protein degradation and regulate autophagy. Aged HSC show an unbalanced scenario where these processes lose their metabolic homeostasis and proteostasis, converging into a status of metabolic and protein stress. Fasting is able to restore at least partially the activity of the UPR ${ }^{E R}$ and proteostasis in aged HSC. ROS: reactive oxygen species; UPR: unfolded protein response. 
Atg1scKO mice, autophagy is deregulated and aged HSC present with reduced quiescence, an increased rate of differentiation biased toward the myeloid lineage, and an increment in OXPHOS rates. In HSC from conditional knockout Atg12 mice, changes in the methylome of essential genes for the formation of autophagosomes have been reported. These changes could be explained by the alterations of metabolite levels caused by the loss of autophagy, such as reduction of S-adenylmethionine (SAM), methyl-donor co-substrate for methylases, and an increment in alpha-ketoglutrate $(\alpha \mathrm{KG})$, a co-factor for demethylases. ${ }^{35,116}$

Intriguingly, it was also reported that a fraction of aged HSC showing high levels of autophagy displayed higher resistance to exhaustion, as demonstrated by secondary transplants in mice. These data suggest that, although aged HSC are apparently phenotypically equal, there might be a subset that maintains the autophagy levels of young HSC and are consequently resilient to aging, preserving their stemness and regenerative potential. ${ }^{35}$

Nowadays, autophagy represents a target for therapeutic interventions in vitro and in vivo, with clinical trials ongoing for several aged-related hematopoietic disorders, such as AML. ${ }^{17,118}$ A number of these autophagy-targeting approaches are based on the use of different MTOR pathway inhibitors ${ }^{119}$ and could offer a potential therapeutic approach to improve healthy aging as well.

\section{Proteostasis role in hematopoietic stem cell aging}

Homeostasis of proteins, also called proteostasis, indicates a situation in which the proteome within the cell reaches an equilibrium by balancing the elimination of misfolded or damaged proteins while preserving at the same time the necessary levels of properly assembled proteins. $^{36}$

Proteostasis relys in part on protein translation, that is, it is directly dependent on ribosomal levels and biogenesis. Transcription of rRNA genes is regulated by the methylation state of the rRNA genes themselves. Interestingly, it has been shown that ribosomal biogenesis is a target of aging with hypo-methylation of rRNA genes and higher levels of transcription in aged HSC. ${ }^{28}$

\section{Cell polarity and aging}

Cell polarity is a universal biological feature, and age seems to be a factor contributing to the loss of cell polarity regulation. ${ }^{120}$ Polarity can be defined as the asymmetric distribution of cellular components, biomolecules and structures within the cells. The number of genes linking polarity and aging is strikingly increasing and, consequently, the number of targets to be investigated in order to ameliorate aging is growing. ${ }^{121}$ For instance, in the baker yeast $S$. cerevisiae, it has been demonstrated that damaged and aggregated proteins are retained in the mother cell establishing an asymmetry that marks which of the cells is aged. The lysine deacetylase Sir2p is responsible for this, since it regulates the ultimate correct folding of actin in order to keep the protein aggregates in the mother (aged) cell. ${ }^{122}$ This mechanism is highly conserved among organisms, as is shown in Drosophila larval and adult stem cells (female germline and intestinal stem cells). Here, proteostasis seems to be, at least in part, reg- ulated by asymmetric division: the shortest life-span daughter inherits the majority of damaged proteins leaving the stem cell daughter as damage-free as possible. ${ }^{123}$ This is particularly important in adult stem cells, since the accumulation of damaged or misfolded proteins can contribute to aging. ${ }^{36,124}$ Asymmetry has been described in HSCs for several proteins such as Cdc42, Dlg, Crumb3, Scribble, H4K16ac, LaminA/C. Polarity of these proteins is characteristic of young HSC and apolarity is more prominent in aged stem cells. . $^{14,15,29,44,125} \mathrm{CD} 71$, the transferrin receptor, and CD53 and CD63, endosomal-associated proteins are also described to be polar and confer asymmetry during division of HSC, being characteristic of the most primitive population of HSC when they are together with the stem marker CD133. ${ }^{126}$

In summary, despite the wide experimental evidence linking loss of cell polarity and the proteostasis decay upon aging, only a few pharmacological approaches have been proposed so far. For example, CASIN was successfully used to recover HSC cell polarity and rejuvenate function of aged stem cells in mice. ${ }^{14}$

\section{Protein degradation systems and folding stress response}

The ubiquitin proteasome system (UPS) is a complex of factors responsible for tagging proteins for signaling or for degradation, and is, therefore, implied in regulating proteostasis in eukaryotic cells. ${ }^{39}$ It has been shown that HSC function can be affected by the absence or malfunction of different UPS system factors, such as E3 ligases or deubiquitinases. This happens in an indirect way, affecting the accumulation of its protein targets, like STAT5, c-myc or Notch, and promoting exacerbated responses. ${ }^{39}$ Moreover, the UPS regulates the degradation of histone modifying enzymes such as HDAC1 and DNMT1. ${ }^{127}$ This means that the epigenetic changes observed might be a consequence of the proteasome decay occurring with aging. ${ }^{128}$

Another system controlling proteostasis is the unfolded protein response that occurs in the endoplasmic reticulum $\left(U P R^{\mathrm{ER}}\right)$. This system preferentially induces apoptosis under ER (endoplasmic reticulum) stress in HSPC compared to closely related progenitors. The biological relevance of this mechanism relys on the elimination by apoptosis of those HSC that are under ER stress due to accumulation of misfolded proteins. ${ }^{129}$ Intimately related with the UPR ${ }^{\mathrm{ER}}$, protein chaperones function by enhancing the correct folding of proteins, and, in turn, protecting against UPR-induced apoptosis. For example, the overexpression of the UPR ${ }^{\mathrm{ER}}$ factor ATF4 and the co-chaperone ERFJ4 in HSC isolated from human cord blood samples confers greater repopulation capacity compared to nonmodified HSC or progenitor cells. ${ }^{129}$

$\mathrm{UPR}^{\mathrm{ER}}$ is up-regulated in intestinal stem cells (ISC) in Drosophila with aging and oxidative stress, which leads to age-related dysplasia and proliferation, ${ }^{130}$ although in other biological organisms, such as $C$. elegans, it promotes longevity. ${ }^{131}$ In mice, UPR ${ }^{\mathrm{ER}}$ is transiently hyper-activated after fasting-re-feeding periods as has been observed by the overexpression of Xbp1, a transcription factor that activates the expression of the UPR ${ }^{\mathrm{ER}}$ components. Furthermore, Xbp1 overexpression leads to hypoglycemia due to an improvement in insulin sensitivity and triggers lipolysis in the liver. ${ }^{132}$ Adaptation to fasting is linked to the UPR ${ }^{\mathrm{ER}}$ system through IRE1 $\alpha$, a protein 
located in the ER that splices Xbp1 mRNA to a form that activates UPR ${ }^{\mathrm{ER}} .{ }^{133}$ Prolonged fasting has a direct effect on the UPR ${ }^{\mathrm{ER}}$ system that influences the self-renewal and differentiation potential of stem cells. ${ }^{134}$ However, the effects of fasting in HSC have so far only been investigated from a different perspective. Prolonged fasting in mice protects HSC and progenitors within the bone marrow after chemotoxicity and promotes balanced hematopoietic regeneration, also in the absence of chemotherapy treatment. The effect of prolonged fasting on HSC is mediated by the low levels of expression of IGF1 (insulin-like growth factor 1) and PKA $\alpha$ in starved mice. ${ }^{135}$

\section{Signaling pathways influencing intrinsic hematopoietic stem cell aging}

Hematopoietic stem cells are located in a very special microenvironment: the bone marrow niche. Communication between the HSC and niche cells is essential for a correct functioning of the whole hematopoietic system. ${ }^{136}$ Hence, it is very important that the signaling pathways between HSC and the niche are functioning correctly throughout the life-span of the organisms. Since we are dealing here with intrinsic alterations of HSC upon aging, we will focus on some examples of signaling factors and pathways within stem cells that are particularly compelling in the context of intrinsic aging.

\section{Tgf- $\beta$ related}

Transcriptome data analysis showed reduced TGF- $\beta$ signaling upon aging in HSC. ${ }^{15,28}$ Sun et al. calculated that TGF- $\beta$-regulated genes were five times more likely to be down-regulated compared to all other genes affected by age. ${ }^{28}$ Among the TGF- $\beta$ downstream genes down-regulated in the study, more than half $(63 \%)$ are related to biological functions that somehow sustain hematopoiesis. ${ }^{43,137}$ In agreement with these findings, scATACseq experiments comparing young and old HSC have shown that TGF- $\beta$ signaling is specifically enriched in young HSC, meaning that they are in a more accessible chromatin context, and consequently, more likely to be expressed in young when compared to old cells. ${ }^{15}$ TGF1$\beta$ signaling is also modulated by the regulation of the stability of its receptor upon aging. Tif1- $\gamma$ (transcription intermediary factor $1 \gamma$ ) regulates TGF1- $\beta$ R turn over via its ubiquitin ligase activity. Tif1- $\gamma$ was reported to be down-regulated in aged HSC, while knock out of Tif1- $\gamma$ promoted premature HSC aging. Consequently, the amount of TGF1- $\beta$ R is higher in aged HSC, making the cells more sensitive to TGF1- $\beta$. $^{45}$

\section{Wnt related}

Wnt signaling has been described to have an important role in aging in different stem cell systems such as muscle stem cells, adult neural stem cells or skin stem cells. ${ }^{138-140}$ In the case of muscle stem cells, with age, canonical Wnt signaling is activated, leading to impaired muscle regeneration and augmented fibrosis. ${ }^{139}$ For adult neural stem cells, it has been shown that aging leads to the overexpression of the Wnt antagonist Dickkopf-1 in order to reduce neurogenesis. When Dickkopf-1 is conditionally ablated, neurogenesis increases. ${ }^{138}$ Regarding skin stem cells, the Wnt antagonist Klotho protects against aging by blocking Wnt signaling. ${ }^{140}$ Wnt family members also have a role in bone marrow function, since different members are expressed in hematopoietic cells and in nonhematopoietic stromal cells. ${ }^{141}$ It has been shown that Wnt signaling is involved in the maintenance of the balance between quiescence and activation of HSC in the bone marrow. ${ }^{40,142}$ In murine HSC, it has been shown that there is an intrinsic increased expression of Wnt5a, shifting the signaling from canonical to non-canonical in aged HSC. ${ }^{44}$ The non-canonical Wnt pathway resulted in Cdc42 activation and induced aging-like phenotypes when Wnt5a was added to young LT-HSC, including apolarity and loss of epigenetic asymmetry at division. ${ }^{15,44}$ In contrast, the haploinsufficiency of Wnt5 showed attenuation of aging phenotypes in old HSC Wnt $5 \mathrm{a}^{+/}$mice. ${ }^{44}$ However, Wnt signaling in aging also has an extrinsic role in the bone marrow since haploinsufficient Wnt5a ${ }^{+/}$ recipient mice regenerate dysfunctional HSC upon secondary transplantation. ${ }^{143}$ This apparent incongruence suggests that Wnt5a might exert both an autocrine and a paracrine effect on HSC.

\section{Notch related}

It is known that Notch is important for the maintenance of murine muscle stem cells since loss of Notch signaling pathway causes impairment in regeneration of muscle. ${ }^{144}$ In fact, the loss of satellite cells that occurs in aged mdx mice, a murine model for the DuchenneMuscular-Disthropy that involves the downregulation of the Notch signaling pathway, can be ameliorated when Notch signaling pathway is restored. ${ }^{145}$ Recently, it was also demonstrated in murine muscle stem cells that ligands of Notch activate p53 during normal regeneration, while this axis is impaired in aged mice leading to cell death due to mitotic catastrophe. ${ }^{146}$

There is also evidence of the implication of Notch2, and not Notch1 in the specific Ventricular-subVentricular Zone (V-SVZ) adult neural stem cells, where it represses cell cycle preserving quiescence. ${ }^{147}$

In the case of the hematopoietic system, there are conflicting results from different studies making this a subject of intense debate (reviewed by Lampreia ${ }^{42}$ and Weber and Calvi $\left.{ }^{148}\right)$. On one hand, the function of Notch signaling in murine HSC has been reported several times as favoring HSC self-renewal and expansion by in vitro stimulation of the Notch pathway, such as the transduction of the active intracellular portion of Notch1 or its targets, Hes $1^{149,150}$ and in vivo by the deletion of a E3 ubiquitin ligase that negatively regulates Notch receptor degradation. ${ }^{151}$ However, most studies based on in vivo loss-of-function models seem to argue against these results, since Notch signaling pathway is not essential for adult hematopoiesis in mice. ${ }^{152,153}$ Notch involvement in human HSC function is also controversial. ${ }^{42}$ On one side, inhibition of Notch by transducing human HSCs with dnMAML1 leads to blockage of maintenance/expansion and T-cell development in vitro, while in vivo engraftment seems not to be affected. ${ }^{154}$ However, by using $\gamma$-secretase inhibitor (DAPT) to inhibit Notch signaling, it has been shown that Notch pathway is important for the repopulation capacity of human HSC. ${ }^{155}$

\section{NF- $\kappa B$ related}

Constitutive NF- $\mathrm{BB}$ activation has been involved in aging in several murine tissues, including bone marrow, ${ }^{156}$ and in age-related myeloid malignancies like AML 
(reviewed by Zhou et al. ${ }^{157}$ ). A very interesting integrative study combining human and mice datasets from young and aged fibroblasts has revealed that NF- $\kappa B$ enforces the aging phenotype and it can be targeted, at least in mice, to revert the aged phenotype. ${ }^{158}$ Also, NF- $\kappa \mathrm{B}$ signaling seems to be activated in aged tissues and it negatively regulates autophagy (reviewed by Salminen et al. ${ }^{159}$ ).

More recently, NF- $\kappa \mathrm{B}$ signaling activation in the hematopoietic system has been studied under inflammation stimuli in mice showing that aged LT-HSC present with an altered myeloid-biased response to inflammation. ${ }^{18}$

In another study, carried out in mice, aged HSC were shown to be failing in down-regulating NF- $\mathrm{KB}$ signaling when an acute inflammatory stimulus occurs, ${ }^{43,160}$ and that this is dependent on the levels of $\operatorname{Rad} 21 /$ cohesin. ${ }^{160}$

3

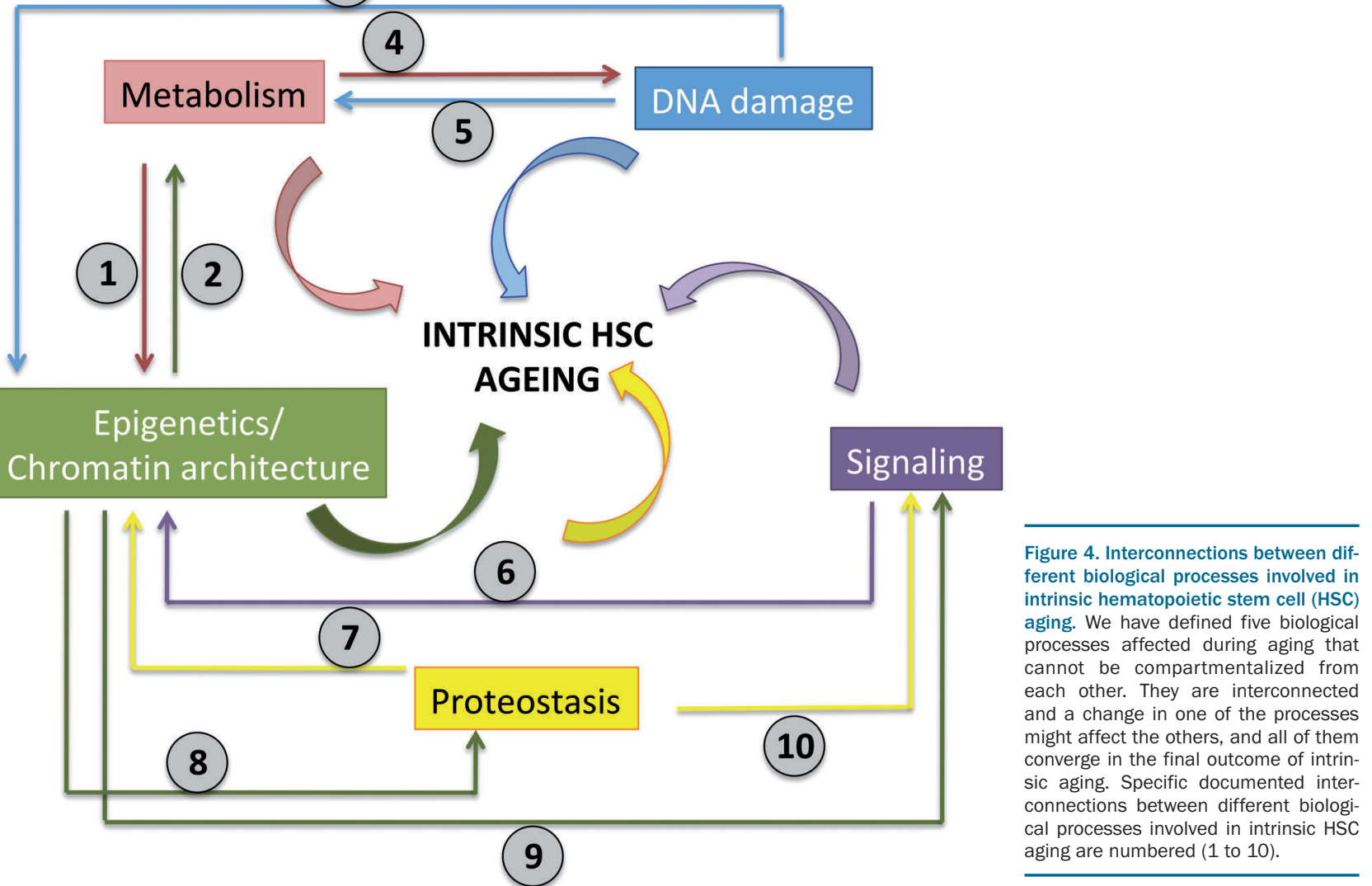

Table 1. Details of documented connections between different biological processes involved in intrinsic hematopoietic stem cell aging.

\begin{tabular}{|c|c|c|}
\hline Connection & Process & Reference \\
\hline 1 & -Change in some metabolite levels affect to the activity of methylases on DNA methylation & (35) \\
\hline 2 & -SIRT7 overexpression in aged HSC, preserves against mitochondrial stress & $(114)$ \\
\hline 3 & -Specific mutations in epigenetic regulators such as DNMT3, TET2 or ASXL2 tend to appear upon ageing & $(53-56)$ \\
\hline 4 & -Accumulation of ROS is related to augmented DNA damage & $(107)$ \\
\hline 5 & $\begin{array}{l}\text {-Potl has a dual role inhibiting ATR-dependent DNA damage repair at telomeres at the same time that it controls } \\
\text { the expression of genes involved in ROS reduction and mTOR signaling }\end{array}$ & $(72)$ \\
\hline 6 & -Wnt non-canonical pathway activates Cdc42 in young HSC inducing ageing-like phenotypes & $(12,44)$ \\
\hline 7 & $\begin{array}{l}\text {-The UPS malfunctions in aged HSC which inhibits the correct degradation of histone modifying enzymes } \\
\text { such as HDACl and DNMT1 }\end{array}$ & $(127,128)$ \\
\hline 8 & $\begin{array}{l}\text {-rRNA genes are hypomethylated in aged HSC which involves increased rRNA transcription, more ribosomal } \\
\text { biogenesis and higher levels of protein translation }\end{array}$ & (28) \\
\hline 9 & $\begin{array}{l}\text {-TGF- } \beta \text { signaling-related genes are enriched in young HSC by ATAC-seq and Wnt signaling-related genes are } \\
\text { enriched in old HSC by ATAC-seq }\end{array}$ & $(15,28)$ \\
\hline 10 & -The UPS malfunctions in aged HSC which inhibits the correct degradation of Notch signaling-related factors & $(39,45)$ \\
\hline
\end{tabular}




\section{Perspectives for rejuvenation and therapeutic strategies}

In this review, we have summarized some of the most recent and relevant information about the intrinsic factors that affect HSC upon aging. In general, we can identify at least five biological processes, which we would define as intrinsic HSC aging mechanisms (Table 1 and Figure 4). DNA damage, metabolism, epigenetics/chromatin architecture, proteostasis, and signaling. However, the cell cannot compartmentalize hermetically each one of these processes. This is the reason why the interconnections between these biological processes will be a key aspect in deciphering the extent of the impact of aging on HSC. For instance, the levels of several metabolites like SAM or ketoglutharate affect the activity of methylases, which in turn have an effect on the epigenetics of the cell..$^{35}$ Investigating and understanding cellular and molecular mechanisms of HSC aging will increase the possibility of defining new pharmacological targets to reduce the negative effects of aging and promote "healthy aging".

We are aware that most aging mechanisms that we have reviewed here have been studied in the murine or in nonhuman systems. Nevertheless, we would like to emphasize the progress achieved so far and the importance of pursuing an integrative research approach, to connect all the aspects affecting stem cell aging. A broader view of this process might indeed be central in bridging the gap to translation in the human system.

\section{Acknowledgment}

This work was supported by the Deutsche Forschungsgemeinschaft (DFG or German Research Foundation) FOR2674 and SFB1074 to MCF and by Ministerio de Ciencia, Innovación y Universidades (Spanish Ministry of Science, Innovation and University) PGC2018-102049-B-I00 and RYC2018-025979-I to MCF.

\section{References}

1. Klepin HD. Myelodysplastic Syndromes and Acute Myeloid Leukemia in the Elderly. Clin Geriatr Med. 2016;32(1):155-173.

2. Akunuru S, Geiger H. Aging, Clonality, and Rejuvenation of Hematopoietic Stem Cells. Trends Mol Med. 2016;22(8):701-712.

3. Preussler JM, Meyer CL, Mau LW, et al. Healthcare Costs and Utilization for Patients Age 50 to 64 Years with Acute Myeloid Leukemia Treated with Chemotherapy or with Chemotherapy and Allogeneic Hematopoietic Cell Transplantation. Biol Blood Marrow Transplant. 2017;23(6):1021-1028.

4. Meyers J, Yu Y, Kaye JA, Davis KL. Medicare fee-for-service enrollees with primary acute myeloid leukemia: an analysis of treatment patterns, survival, and healthcare resource utilization and costs. Appl Health Econ Health Policy. 2013;11(3):275-286.

5. Chandel NS, Jasper H, Ho TT, Passegue E. Metabolic regulation of stem cell function in tissue homeostasis and organismal ageing. Nat Cell Biol. 2016;18(8):823-832.

6. Singh AK, Althoff MJ, Cancelas JA. Signaling Pathways Regulating Hematopoietic Stem Cell and Progenitor Aging. Current Stem Cell Rep. 2018;4(2):166-181

7. Rossi DJ, Bryder D, Zahn JM, et al. Cell intrinsic alterations underlie hematopoietic stem cell aging. Proc Natl Acad Sci U S A. 2005;102(26):9194-9199.

8. Sudo K, Ema H, Morita Y, Nakauchi H. AgeAssociated Characteristics of Murine Hematopoietic Stem Cells. J Exp Med. 2000;192(9):1273-1280

9. Pang WW, Price EA, Sahoo D, et al. Human bone marrow hematopoietic stem cells are increased in frequency and myeloid-biased with age. Proc Natl Acad Sci U S A. 2011;108(50):20012-20017.

10. Vas V, Senger K, Dorr K, Niebel A, Geiger H. Aging of the microenvironment influences clonality in hematopoiesis. PLoS One. 2012;7(8):e42080.

11. Ergen AV, Boles NC, Goodell MA. Rantes/Ccl5 influences hematopoietic stem cell subtypes and causes myeloid skewing. Blood. 2012;119(11):2500-2509.
12. Guidi N, Sacma M, Standker L, et al. Osteopontin attenuates aging-associated phenotypes of hematopoietic stem cells EMBO J. 2017;36(7):840-853

13. Beerman I, Maloney WJ, Weissmann IL, Rossi DJ. Stem cells and the aging hematopoietic system. Curr Opin Immunol. 2010;22(4):500-506

14. Florian MC, Dorr K, Niebel A, et al. Cdc42 activity regulates hematopoietic stem cell aging and rejuvenation. Cell Stem Cell. 2012;10(5):520-530

15. Florian MC, Klose M, Sacma M, et al. Aging alters the epigenetic asymmetry of HSC division. PLoS Biol. 2018;16(9):e2003389.

16. Signer RA, Montecino-Rodriguez E, Witte ON, McLaughlin J, Dorshkind K. Age-related defects in B lymphopoiesis underlie the myeloid dominance of adult leukemia. Blood. 2007;110(6):1831-1839.

17. Yamamoto R, Wilkinson AC, Ooehara J, et al. Large-Scale Clonal Analysis Resolves Aging of the Mouse Hematopoietic Stem Cell Compartment. Cell Stem Cell. 2018;22(4):600-607.

18. Mann M, Mehta A, de Boer CG, et al. Heterogeneous Responses of Hematopoietic Stem Cells to Inflammatory Stimuli Are Altered with Age. Cell Rep. 2018;25(11):2992-3005.

19. Lopez-Otin C, Blasco MA, Partridge L, Serrano M, Kroemer G. The hallmarks of aging. Cell. 2013;153(6):1194-1217.

20. Beerman I, Seita J, Inlay MA, Weissman IL Rossi DJ. Quiescent hematopoietic stem cells accumulate DNA damage during aging that is repaired upon entry into cell cycle. Cell Stem Cell. 2014;15(1):37-50.

21. Flach J, Bakker ST, Mohrin M, et al. Replication stress is a potent driver of functional decline in ageing haematopoietic stem cells. Nature. 2014;512(7513):198-202.

22. Akunuru S, Geiger H. Aging, Clonality, and Rejuvenation of Hematopoietic Stem Cells. Trends Mol Med. 2016;22(8):701-712

23. Chen J, Kao YR, Sun D, et al. Myelodysplastic syndrome progression to acute myeloid leukemia at the stem cell level. Nat Med. 2019;25(1):103-110

24. Steensma DP, Bejar R, Jaiswal S, et al. Clonal hematopoiesis of indeterminate potential and its distinction from myelodysplastic syndromes. Blood. 2015;126(1):9-16

25. Jaiswal S, Natarajan P, Silver AJ, et al. Clonal Hematopoiesis and Risk of Atherosclerotic Cardiovascular Disease. N Engl J Med. 2017;377(2):111-121.

26. Yu VWC, Yusuf RZ, Oki T, et al. Epigenetic Memory Underlies Cell-Autonomous Heterogeneous Behavior of Hematopoietic Stem Cells. Cell. 2016;167(5):1310-1322

27. Beerman I, Bock C, Garrison BS, et al Proliferation-dependent alterations of the DNA methylation landscape underlie hematopoietic stem cell aging. Cell Stem Cell. 2013;12(4):413-425.

28. Sun $D$, Luo $M$, Jeong $M$, et al. Epigenomic Profiling of Young and Aged HSCs Reveals Concerted Changes during Aging that Reinforce Self-Renewal. Cell Stem Cell. 2014;14(5):673-688

29. Grigoryan A, Guidi N, Senger K, et al LaminA/C regulates epigenetic and chromatin architecture changes upon aging of hematopoietic stem cells. Genome Biol. 2018;19(1):189.

30. Maryanovich M, Zahalka AH, Pierce $\mathrm{H}$, et al. Adrenergic nerve degeneration in bone marrow drives aging of the hematopoietic stem cell niche. Nat Med. 2018;24(6):782791.

31. Ito K, Suda T. Metabolic requirements for the maintenance of self-renewing stem cells. Nat Rev Mol Cell Biol. 2014;15(4):243-256.

32. Verovskaya EV, Dellorusso PV, Passegué E. Losing Sense of Self and Surroundings: Hematopoietic Stem Cell Aging and Leukemic Transformation. Trends Mol Med. 2019;25(6):494-515.

33. He C, Klionsky DJ. Regulation mechanisms and signaling pathways of autophagy. Annu Rev Genet. 2009:43:67-93.

34. Kaushik S, Cuervo AM. The coming of age of chaperone-mediated autophagy. Nat Rev Mol Cell Biol. 2018;19(6):365-381

35. Ho TT, Warr MR, Adelman ER, et al Autophagy maintains the metabolism and function of young and old stem cells. Nature. 2017;543(7644):205-210.

36. Vilchez D, Simic MS, Dillin A. Proteostasis and aging of stem cells. Trends Cell Biol. 2014:24(3):161-170

37. Noormohammadi A, Calculli G, GutierrezGarcia R, Khodakarami A, Koyuncu S, 

novel effector for $\mathrm{Cdc} 42 \mathrm{Hs}$, is implicated in the reorganization of the actin cytoskeleton and in the formation of filopodia. EMBO J. 1998;17(22):6527-6540.

95. Kong S, Zhang Y. Deciphering Hi-C: from 3D genome to function. Cell Biol Toxicol. 2019;35(1):15-32.

96. Rowley MJ, Corces VG. Organizational principles of 3D genome architecture. Nat Rev Genet. 2018;19(12):789-800.

97. Galeev R, Larsson J. Cohesin in haematopoiesis and leukaemia. Curr Opin Hematol. 2018;25(4):259-265.

98. Viny AD, Ott CJ, Spitzer B, et al. Dosedependent role of the cohesin complex in normal and malignant hematopoiesis. J Exp Med. 2015;212(11):1819-1832.

99. Choudry FA, Frontini M. Epigenetic Control of Haematopoietic Stem Cell Aging and Its Clinical Implications. Stem Cells Int. 2016;2016:5797521.

100. Benayoun BA, Pollina EA, Singh PP, et al. Remodeling of epigenome and transcriptome landscapes with aging in mice reveals widespread induction of inflammatory responses. Genome Res. 2019;29(4):697709 .

101. Cheung P, Vallania F, Warsinske HC, et al. Single-Cell Chromatin Modification Profiling Reveals Increased Epigenetic Variations with Aging. Cell. 2018;173(6): 1385-1397.

102. Burman B, Zhang ZZ, Pegoraro G, Lieb JD, Misteli $\mathrm{T}$. Histone modifications predispose genome regions to breakage and translocation. Genes Dev. 2015;29(13):1393-1402.

103. Kirschner K, Chandra T, Kiselev V, et al. Proliferation Drives Aging-Related Functional Decline in a Subpopulation of the Hematopoietic Stem Cell Compartment. Cell Rep. 2017;19(8):1503-1511.

104. Bigarella CL, Liang R, Ghaffari S. Stem cells and the impact of ROS signaling. Development. 2014;141(22):4206-4218.

105. Norddahl GL, Pronk CJ, Wahlestedt M, et al. Accumulating mitochondrial DNA mutations drive premature hematopoietic aging phenotypes distinct from physiological stem cell aging. Cell Stem Cell. 2011;8(5):499-510.

106. Jang YY, Sharkis SJ. A low level of reactive oxygen species selects for primitive hematopoietic stem cells that may reside in the low-oxygenic niche. Blood. 2007;110(8): 3056-3063.

107. Rimmele P, Liang R, Bigarella CL, et al. Mitochondrial metabolism in hematopoietic stem cells requires functional $\mathrm{FOXO}$. EMBO Rep. 2015;16(9):1164-1176.

108. Tothova Z, Kollipara R, Huntly BJ, et al. FoxOs are critical mediators of hematopoietic stem cell resistance to physiologic oxidative stress. Cell. 2007;128(2):325-339.

109. Warr MR, Binnewies M, Flach J, et al. FOXO3A directs a protective autophagy program in haematopoietic stem cells. Nature. 2013;494(7437):323-327.

110. Brown K, Xie S, Oiu X, et al. SIRT3 reverses aging-associated degeneration. Cell Rep. 2013;3(2):319-327.

111. Trifunovic A, Wredenberg A, Falkenberg M, et al. Premature ageing in mice expressing defective mitochondrial DNA polymerase. Nature. 2004:429(6990):417-423.

112. Chen F, Liu Y, Wong NK, Xiao J, So KF. Oxidative Stress in Stem Cell Aging. Cell Transplant. 2017;26(9):1483-1495.

113. Oh J, Lee YD, Wagers AJ. Stem cell aging: mechanisms, regulators and therapeutic opportunities. Nat Med. 2014;20(8):870-880.

114. Mohrin M, Shin J, Liu Y, et al. Stem cell aging. A mitochondrial UPR-mediated metabolic checkpoint regulates hematopoi- etic stem cell aging. Science. 2015;347(6228): 1374-1377.

115. Rubinsztein David C, Mariño G, Kroemer G. Autophagy and Aging. Cell. 2011;146(5): 682-695.

116. Schvartzman JM, Thompson CB, Finley LWS. Metabolic regulation of chromatin modifications and gene expression. J Cell Biol. 2018;217(7):2247-2259.

117. Auberger P, Puissant A. Autophagy, a key mechanism of oncogenesis and resistance in leukemia. Blood. 2017;129(5):547-552

118. Rothe K, Porter V, Jiang X. Current Outlook on Autophagy in Human Leukemia: Foe in Cancer Stem Cells and Drug Resistance, Friend in New Therapeutic Interventions. Int J Mol Sci. 2019;20(3).

119. Willems L, Chapuis N, Puissant A, et al. The dual mTORC1 and mTORC2 inhibitor AZD8055 has anti-tumor activity in acute myeloid leukemia. Leukemia. 2012;26(6): 1195-1202.

120. Macara IG, Mili S. Polarity and differential inheritance--universal attributes of life? Cell. 2008;135(5):801-812.

121. Budovsky A, Fraifeld VE, Aronov S. Linking cell polarity, aging and rejuvenation. Biogerontology. 2011:12(2):167-175

122. Liu B, Larsson L, Caballero A, et al. The polarisome is required for segregation and retrograde transport of protein aggregates. Cell. 2010;140(2):257-267.

123. Bufalino MR, DeVeale B, van der Kooy D. The asymmetric segregation of damaged proteins is stem cell-type dependent. J Cell Biol. 2013;201(4):523-530

124. Yamashita YM, Yuan H, Cheng J, Hunt AJ. Polarity in stem cell division: asymmetric stem cell division in tissue homeostasis. Cold Spring Harb Perspect Biol. 2010;2(1):a001313

125. Mohr J, Dash BP, Schnoeder TM, et al. The cell fate determinant Scribble is required for maintenance of hematopoietic stem cell function. Leukemia. 2018;32(5):1211-1221.

126. Beckmann J, Scheitza S, Wernet P, Fischer JC, Giebel B. Asymmetric cell division within the human hematopoietic stem and progenitor cell compartment: identification of asymmetrically segregating proteins. Blood. 2007;109(12):5494-5501.

127.Zou C, Mallampalli RK. Regulation of histone modifying enzymes by the ubiquitinproteasome system. Biochim Biophys Acta. 2014:1843(4):694-702

128. Tomaru U, Takahashi S, Ishizu A, et al. Decreased proteasomal activity causes agerelated phenotypes and promotes the development of metabolic abnormalities. Am J Pathol. 2012;180(3):963-972.

129. van Galen P, Kreso A, Mbong N, et al. The unfolded protein response governs integrity of the haematopoietic stem-cell pool during stress. Nature. 2014:510(7504):268-272.

130.Wang L, Zeng X, Ryoo HD, Jasper H. Integration of UPRER and oxidative stress signaling in the control of intestinal stem cell proliferation. PLoS Genet. 2014;10(8) :e1004568.

131. Henis-Korenblit S, Zhang P, Hansen M, et al Insulin/IGF-1 signaling mutants reprogram ER stress response regulators to promote longevity. Proc Natl Acad Sci U S A. 2010;107(21):9730-9735.

132.Deng Y, Wang ZV, Tao C, et al. The Xbp1s/GalE axis links ER stress to postprandial hepatic metabolism. J Clin Invest. 2013;123(1):455-468.

133. Shao M, Shan B, Liu Y, et al. Hepatic IRE1CE \pm regulates fasting-induced metabolic adaptive programs through the XBP1sPPAROE \pm axis signalling. Nat Commun.
2014;5:3528.

134. Chaube R. Can UPR integrate fasting and stem cell regeneration? Front Chem. 2015;3:5.

135. Cheng CW, Adams GB, Perin L, et al. Prolonged fasting reduces IGF-1/PKA to promote hematopoietic-stem-cell-based regeneration and reverse immunosuppression. Cell Stem Cell. 2014;14(6):810-823.

136. Wei Q, Frenette PS. Niches for Hematopoietic Stem Cells and Their Progeny. Immunity. 2018;48(4):632-648.

137. Challen GA, Boles NC, Chambers SM Goodell MA. Distinct hematopoietic stem cell subtypes are differentially regulated by TGF-beta1. Cell Stem Cell. 2010;6(3):265278

138. Seib DR, Corsini NS, Ellwanger K, et al. Loss of Dickkopf-1 restores neurogenesis in old age and counteracts cognitive decline. Cell Stem Cell. 2013;12(2):204-214.

139. Brack AS, Conboy MJ, Roy S, et al. Increased Wnt signaling during aging alters muscle stem cell fate and increases fibrosis. Science. 2007;317(5839):807-810.

140. Liu H, Fergusson MM, Castilho RM, et al. Augmented Wnt signaling in a mammalian model of accelerated aging. Science. 2007;317(5839):803-806

141. Malhotra S, Kincade PW. Wnt-related molecules and signaling pathway equilibrium in hematopoiesis. Cell Stem Cell. 2009;4(1):2736.

142. Dijksterhuis IP, Petersen I, Schulte G WNT/Frizzled signalling: receptor-ligand selectivity with focus on FZD-G protein signalling and its physiological relevance: IUPHAR Review 3. Br J Pharmacol. 2014;171(5):1195-1209.

143. Schreck C, Istvanffy R, Ziegenhain C, et al. Niche WNT5A regulates the actin cytoskeleton during regeneration of hematopoietic stem cells. J Exp Med. 2017:214(1):165-181.

144. Conboy IM, Conboy MJ, Wagers AJ, Girma ER, Weissman IL, Rando TA. Rejuvenation of aged progenitor cells by exposure to a young systemic environment. Nature. 2005;433(7027):760-764.

145. Jiang C, Wen $Y$, Kuroda $K$, Hannon $K$, Rudnicki MA, Kuang S. Notch signaling deficiency underlies age-dependent depletion of satellite cells in muscular dystrophy. Dis Model Mech. 2014;7(8):997-1004.

146. Liu L, Charville GW, Cheung TH, et al Impaired Notch Signaling Leads to a Decrease in p53 Activity and Mitotic Catastrophe in Aged Muscle Stem Cells. Cell Stem Cell. 2018;23(4):544-556.

147. Engler A, Rolando C, Giachino C, et al Notch2 Signaling Maintains NSC Quiescence in the Murine VentricularSubventricular Zone. Cell Rep. 2018;22(4): 992-1002.

148. Weber JM, Calvi LM. Notch signaling and the bone marrow hematopoietic stem cell niche. Bone. 2010;46(2):281-285

149. Calvi LM, Adams GB, Weibrecht KW, et al. Osteoblastic cells regulate the haematopoietic stem cell niche. Nature. 2003;425(6960): 841-846.

150. Karanu FN, Murdoch B, Gallacher L, et al The Notch Ligand Jagged-1 Represents a Novel Growth Factor of Human Hematopoietic Stem Cells. J Exp Med. 2000;192(9):1365-1372.

151. Rathinam C, Matesic LE, Flavell RA. The E3 ligase Itch is a negative regulator of the homeostasis and function of hematopoietic stem cells. Nat Immunol. 2011;12(5):399 407.

152. Maillard I, Koch U, Dumortier A, et al Canonical notch signaling is dispensable for 
the maintenance of adult hematopoietic stem cells. Cell Stem Cell. 2008;2(4):356366.

153. Mancini SJ, Mantei N, Dumortier A, Suter U, MacDonald HR, Radtke F. Jagged1dependent Notch signaling is dispensable for hematopoietic stem cell self-renewal and differentiation. Blood. 2005;105(6):23402342.

154. Benveniste P, Serra P, Dervovic D, et al. Notch signals are required for in vitro but not in vivo maintenance of human hematopoietic stem cells and delay the appearance of multipotent progenitors. Blood. 2014;123(8):1167-1177.
155. Anjos-Afonso F, Currie E, Palmer HG, Foster KE, Taussig DC, Bonnet D. CD34(-) cells at the apex of the human hematopoietic stem cell hierarchy have distinctive cellular and molecular signatures. Cell Stem Cell. 2013;13(2):161-174.

156. Spencer NF, Poynter ME, Im SY, Daynes RA. Constitutive activation of NF-kappa B in an animal model of aging. Int Immunol. 1997;9(10):1581-1588.

157.Zhou J, Ching YQ, Chng WJ. Aberrant nuclear factor-kappa B activity in acute myeloid leukemia: from molecular pathogenesis to therapeutic target. Oncotarget. 2015;6(8):5490-5500.
158. Adler AS, Sinha S, Kawahara TL, Zhang JY Segal E, Chang HY. Motif module map reveals enforcement of aging by continual NF-kappaB activity. Genes Dev. 2007;21(24) 3244-3257.

159. Salminen A, Hyttinen JM, Kauppinen A Kaarniranta K. Context-Dependent Regulation of Autophagy by IKK-NF-kB Signaling: Impact on the Aging Process. Int J Cell Biol. 2012;2012:849541.

160. Chen Z, Amro EM, Becker F, et al. Cohesinmediated NF-kB signaling limits hematopoietic stem cell self-renewal in aging and inflammation. J Exp Med. 2019;216(1):152175. 